\title{
Stability and Agreement of a Microtransducer and an Air-Filled Balloon Esophageal Catheter in the Monitoring of Esophageal Pressure
}

\author{
Renan Maloni Augusto, André Luis Pereira Albuquerque MD PhD, Thomas Jaeger, \\ Carlos Roberto Ribeiro de Carvalho MD PhD, and Pedro Caruso MD PhD
}

\begin{abstract}
BACKGROUND: The use of esophageal catheters with microtransducer promises advantages over traditional catheters with air-filled balloons. However, performance comparisons between these 2 types of catheters are scarce and incomplete. METHODS: A catheter with a 9.5-cm air-filled balloon at the distal tip and a catheter with a microtransducer mounted within a flexible silicone rubber were tested in vitro and in vivo. In vitro, the response times of both catheters were compared, and the drift of the baseline pressure of the microtransducer catheter was evaluated over a 6-h period. In vivo, 11 healthy volunteers had both catheters inserted, and the drift of the baseline esophageal pressure was measured over a 3-h period. Also, the correlation and agreement of the baseline and changes in the esophageal pressure of both catheters were evaluated. RESULTS: In vitro, the microtransducer catheter had a response time significantly higher $(262 \times 114 \mathrm{~Hz}$, $P<.01)$ and a good pressure stability, with a mean baseline pressure drift of $1.4 \mathrm{~cm} \mathrm{H}_{2} \mathrm{O}$. In vivo, both catheters presented a small and similar baseline esophageal pressure drift $(P=0.08)$. For measurements of baseline and changes in esophageal pressure, the correlation and agreement between the catheters were poor, with a large bias between them. CONCLUSIONS: The catheter with the microtransducer had a small baseline pressure drift, similar to the air-filled balloon catheter. The low agreement between the catheters does not allow the microtransducer catheter to be used as a surrogate for the traditional air-filled balloon catheter. Key words: catheterization /methods; esophagus/physiology; humans; manometry/methods; pressure; respiratory muscles/physiology. [Respir Care 2017;62(2):215-221. (C) 2017 Daedalus Enterprises]
\end{abstract}

\section{Introduction}

The measurement of esophageal pressure has frequently been employed in animal and human research. ${ }^{1,2}$ Esophageal pressure measurement has been proposed in the clin-

\footnotetext{
The authors are affiliated with the Pulmonary Division, Heart Institute (InCor), Hospital das Clínicas, Faculdade de Medicina da Universidade de São Paulo, São Paulo, Brazil. Dr Caruso is also affiliated with the Intensive Care Unit, AC Camargo Cancer Center, São Paulo, Brazil.

This study was supported and funded by Fundação de Amparo a Pesquisa do Estado de São Paulo (FAPESP) Grant 2013/02874-8. FAPESP is a governmental non-profit agency. The authors have disclosed no conflicts of interest.

Mr Maloni presented a version of this paper at the International Congress of the European Respiratory Society, held September 6-10, 2014, in Munich, Germany.
}

ical arena to guide mechanical ventilation ${ }^{1,3,4}$, to monitor respiratory muscle activity and patient-ventilator synchrony, and to guide the weaning process. ${ }^{1}$ Due to its importance in research and increasing clinical application, a working group summarized the current knowledge on esophageal pressure measurement and suggested the methods in which these measurements should be used in critically ill patients. ${ }^{1}$ Probably due to the lack of studies, no recommendation about the type of esophageal catheter was stated.

\footnotetext{
Correspondence: Pedro Caruso MD PhD, Avenida Dr Enéas de Carvalho Aguiar, 45. $5^{\circ}$ andar, bloco 2, sala 1, 05403-900 São Paulo-SP, Brazil. E-mail: pedro.caruso@hc.fm.usp.br.
}

DOI: $10.4187 /$ respcare. 04849 
Esophageal pressure can be measured through catheters using liquid-filled balloons, ${ }^{5}$ air-filled balloons, or pressure microtransducers. ${ }^{1,6,7}$ Microtransducer catheters have advantages, such as an absence of air leaks, greater comfort than air-filled balloon catheters, ${ }^{8}$ and easier placement in the correct esophageal position because the pressures are continuously shown during the insertion. Another possible advantage of microtransducer catheters over air-filled balloon catheters during mechanical ventilation is the decrease of cardiac artifacts affecting the esophageal pressure recording, which is most marked in the supine position. Cardiac artifacts are likely to result from direct pressure from overlying mediastinal structures on the air-filled balloon, but the small microtransducer allows it to be placed in the correct esophageal zone but away from pulsatile mediastinal structures. ${ }^{6}$ These advantages could make the microtransducer catheters more suitable for use during long periods, as in critically ill patients during mechanical ventilation.

Air-filled balloon and microtransducer catheters have been compared in vitro and in vivo. $6,8-10$ However, the in vivo studies were performed with the catheters inserted during short periods, ${ }^{6,8-10}$ and clinical use during mechanical ventilation will require knowledge of the catheters' properties during long periods. In vivo, microtransducer catheters are better tolerated than air-filled balloon catheters and were considered accurate to measure relative pressure changes ${ }^{6,8-10}$ but show lower repeatability ${ }^{10}$ and higher baseline drift, making them unreliable for absolute pressure measurements. ${ }^{6,8}$ In clinical use, esophageal catheters must have no baseline drift, measure pressure accurately (especially transpulmonary pressure), and the ability to detect changes in esophageal pressure in order to calculate variables such as work of breathing or pressure-time product.

If current microtransducer catheters prove to agree with the ubiquitous air-filled balloon catheters, due to their advantages, microtransducer catheters would be ideal for clinical use mainly because they are easier to place in the correct esophageal position and easier to handle during the long mechanical ventilation periods because air leaks are not an issue. The objective of the present study was to compare the baseline esophageal pressure drift between microtransducer catheters and air-filled balloon catheters during a 3-h in vivo study. Also, we evaluated the agreement of the absolute esophageal pressure and changes in the esophageal pressure measured with microtransducer and air-filled balloon catheters.

\section{Methods}

We designed an in vivo study to compare air-filled balloon catheters with microtransducer catheters. Eleven healthy volunteers participated in the study. All volunteers

\section{QUICK LOOK}

\section{Current knowledge}

Esophageal pressure measurement is important in the experimental arena and has been proposed to guide mechanical ventilation and weaning. Esophageal pressure can be measured through different catheters, and catheters with microtransducers have attractive advantages over customary esophageal catheters with air-filled balloons, such as the absence of air leaks, greater comfort, and easier placement in the correct esophageal position.

\section{What this paper contributes to our knowledge}

The esophageal catheter with a microtransducer had a small baseline esophageal pressure drift, similar to that of the air-filled balloon catheter. The low agreement between the catheters does not allow the microtransducer catheter to be used as a surrogate for the customary air-filled balloon catheter because the normal ranges established with air-filled balloon catheter may not be strictly applicable.

were medical students, physicians, or respiratory therapists from the Hospital das Clínicas da Faculdade de Medicina da USP ranging from 21 to $47 \mathrm{y}$ old; 9 volunteers were male. The study was approved by the local ethical committee (CapPesq), and informed consent was obtained from each volunteer.

\section{Catheters}

The air-filled balloon catheter was an 86-cm length catheter with a balloon of $9.5-\mathrm{cm}$ length (Adult Esophageal Balloon Catheter Set, CooperSurgical, Trumbull, Connecticut). The microtransducer catheter was $120 \mathrm{~cm}$ in length (2-mm external diameter) with 2 transducers $(5 \mathrm{~mm}$ long by $2 \mathrm{~mm}$ wide) mounted within a flexible silicone rubber catheter, 5 and $35 \mathrm{~cm}$ from the distal tip (Digitimer, Gaeltec, Dunvegan, Isle of Skye, United Kingdom).

According to the manufacturer's instructions, $1 \mathrm{~h}$ before the experiment, the microtransducer catheter was plugged into its amplifier (S7d, Gaeltec, Dunvegan, Isle of Skye, United Kingdom) and submerged into a column of water at controlled room temperature $\left(24^{\circ} \mathrm{C}\right)$. The amplifier acted as an interface between the microtransducer catheter and our acquisition system (ADS 1000, Lynx Eletrônica, São Paulo, Brazil) and was used exclusively as an interface because the analogical signal coming from the microtransducer was not amplified.

A 3-point calibration $\left(0,20\right.$, and $\left.40 \mathrm{~cm} \mathrm{H}_{2} \mathrm{O}\right)$ of the pressure transducer connected to the air-filled balloon was 
performed using a water $\mathrm{U}$ column. The microtransducer calibration was performed by placing the catheter within a 70-cm water column at room temperature, and a 3-point calibration was performed $\left(0,20\right.$, and $\left.40 \mathrm{~cm} \mathrm{H}_{2} \mathrm{O}\right)$. The linearity of amplifier output of the microtransducer and the linearity of the air-filled balloon pressure transducer were recorded during the calibrations. The calibrations were repeated at the beginning of each day.

\section{Study Design}

The air-filled balloon catheter was inserted while deflated until its distal tip was $65 \mathrm{~cm}$ from the nares and within the stomach. Then the air-filled balloon catheter was withdrawn approximately $20 \mathrm{~cm}$ to lie in the midesophagus. ${ }^{1,11}$ The balloon was filled with $4.0 \mathrm{~mL}$, and $3.5 \mathrm{~mL}$ were withdrawn. The remaining $0.5 \mathrm{~mL}$ was continually checked throughout the study.

After the air-filled balloon catheter was in place, the microtransducer catheter was inserted through the same nostril while the 2 transducer curves were continually depicted in a personal computer screen. The microtransducer catheter was advanced until the esophageal and gastric transducers showed a curve with an upward inflection during the inspiration (gastric pressure), and then the catheter was gently withdrawn until the proximal transducer showed a curve with a downward inflection during the inspiration (esophageal pressure). The correct esophageal position of both catheters was checked by the Baydur maneuver. ${ }^{12} \mathrm{We}$ inserted the microtransducer catheter to confirm that its esophageal sensor lay at the middle of the air-filled balloon.

The volunteers were kept in a semi-recumbent position for $3 \mathrm{~h}$ with both catheters inserted. After the volunteers were comfortable and the esophageal pressure was stable, the esophageal pressure was continuously recorded.

To measure the baseline esophageal pressure drift, a reference period was defined as the mean esophageal pressure values from the 2 nd to the 5 th minute. The initial period was defined as the mean esophageal pressure of the 25 th to 30th minute. The final period was defined as the mean esophageal pressure of the last $5 \mathrm{~min}$ of the 3-h period. Baseline esophageal pressure was defined as the esophageal pressure at the end of an expiration. Artifacts in the esophageal pressure due to movement or swallowing were discarded. During the final period, the volunteers were asked to perform 5 sniff maneuvers with a 30 -s interval among them.

\section{Response Time Measurement}

The response times of both catheters were evaluated by inserting the tip of the air-filled balloon or the microtrans-

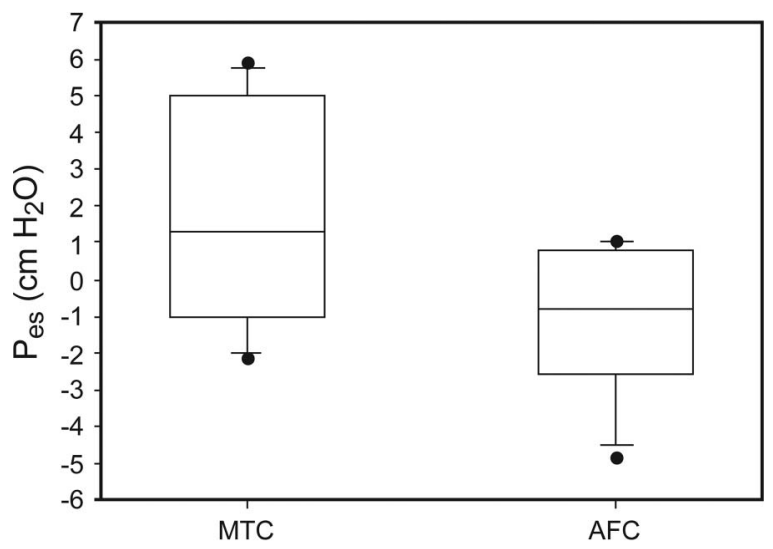

Fig. 1. In vivo baseline esophageal pressure drift at the end of a 3-h period for the microtransducer catheter (MTC) and air-filled balloon catheter (AFC). Boxes denote the first and third quartiles, and center lines show the median.

ducer catheter into a latex balloon, which was inflated to $40 \mathrm{~cm} \mathrm{H}_{2} \mathrm{O}$ and then burst. The frequency response was the time taken for the recorded pressure to fall between 90 and $10 \%$ of the initial pressure and was expressed in Hz. ${ }^{11}$

\section{Acquisition System}

The acquisition system was an analog to digital converter (ADS 1000), sequentially connected to a personal computer running software designed to acquire and analyze the data (AqDados and AqDAnalysis 7.2, Lynx Eletrônica, São Paulo, Brazil). Data were sampled at 1.000 $\mathrm{Hz}$.

\section{Statistical Analyses}

Categorical data are presented as absolute number and percentage. Continuous data are presented as median and interquartile range. The pressure drift and linearity were compared using the Wilcoxon test. The agreement between the values measured by both catheters was evaluated using the Bland-Altman test. ${ }^{13} \mathrm{~A} P$-value of $\leq .05$ was considered statistically significant. All statistical analyses were performed using SPSS 20.0 (SPSS, Chicago, Illinois).

\section{Results}

\section{In Vivo Esophageal Pressure Drift}

After $3 \mathrm{~h}$, both catheters presented a small and similar baseline esophageal pressure drift $\left(1.3 \mathrm{~cm} \mathrm{H}_{2} \mathrm{O}\right.$ for microtransducer vs $-0.8 \mathrm{~cm} \mathrm{H}_{2} \mathrm{O}$ for air-filled balloon, $P=.08$ ) (Fig. 1). 

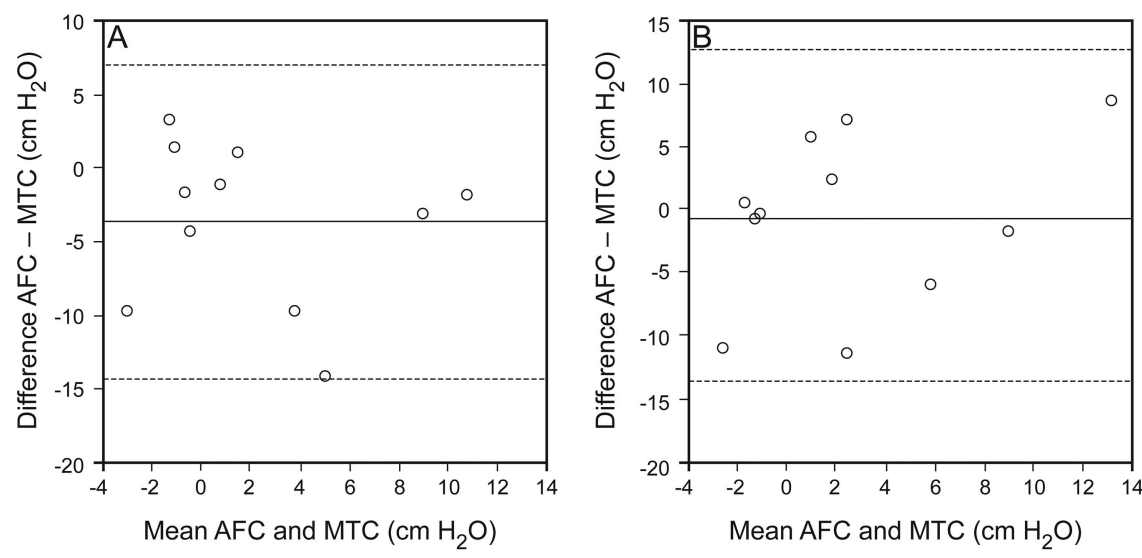

Fig. 2. In vivo correlation and agreement of absolute esophageal pressure during quiet breathing. Correlation of the initial baseline esophageal pressure between the microtransducer catheter (MTC) and air-filled balloon catheter (AFC) during quiet breathing at the beginning of the 3-h period $(A)$. Agreement of the final baseline esophageal pressure between catheters during quiet breathing at the end of the 3 - $h$ period $(B)$. Center lines denote the mean, and outer lines are $\pm 2 S D$.
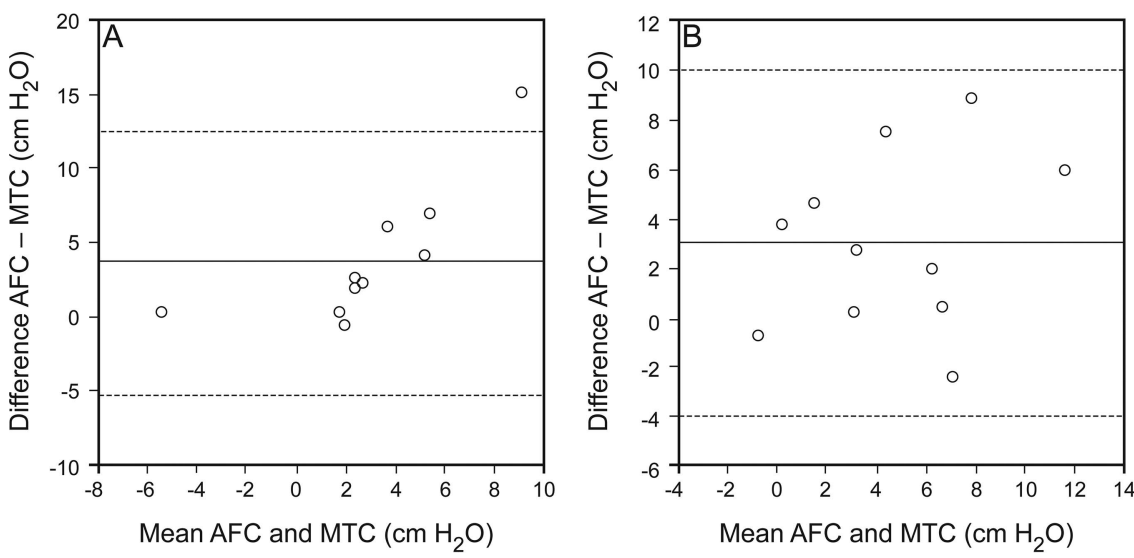

Fig. 3. In vivo correlation and agreement of esophageal pressure change during quiet breathing. Correlation of pressure change between the microtransducer catheter (MTC) and air-filled balloon catheter (AFC) during quiet breathing at the beginning of the 3-h period (A). Agreement of the pressure change between catheters during quiet breathing at the end of the 3-h period (B). Center lines denote the mean, and outer lines are $\pm 2 \mathrm{SD}$.

\section{In Vivo Baseline Esophageal Pressure Agreement}

At the beginning of the measurement, the analysis of agreement of the esophageal baseline pressure showed a poor agreement, with a bias of $-3.6 \mathrm{~cm} \mathrm{H}_{2} \mathrm{O}$ and limits of agreement of -14.3 to $7.0 \mathrm{~cm} \mathrm{H}_{2} \mathrm{O}$ (Fig. 2A). At the end of the third hour of the measurement, the bias was $-0.5 \pm 6.7 \mathrm{~cm} \mathrm{H}_{2} \mathrm{O}$, still with large limits of agreement of -13.7 to $12.7 \mathrm{~cm} \mathrm{H}_{2} \mathrm{O}$ (Fig. 2B).

\section{In Vivo Esophageal Pressure Change Agreement}

At the initial $5 \mathrm{~min}$ of the measurement, the analysis of agreement of the change of the esophageal pressure between the catheters showed a poor agreement with a bias of -3.7 , with limits of agreement of -5.1 to $12.6 \mathrm{~cm} \mathrm{H}_{2} \mathrm{O}$ (Fig. 3A). At the end of the third hour of the measurement, the bias was 3.0 with limits of agreement of -3.9 to $9.9 \mathrm{~cm} \mathrm{H}_{2} \mathrm{O}$ (Fig. 3B).

\section{In Vivo Esophageal Pressure Change During Sniff Maneuvers}

In all measurements during a sniff maneuver, the microtransducer catheter depicted higher values (in absolute value) than the air-filled balloon catheter. The analysis of agreement between the catheters showed a poor agreement, with a bias of $-50.6 \mathrm{~cm} \mathrm{H}_{2} \mathrm{O}(95 \%$ CI -60.6 to -40.6 ) (Fig. 4). 


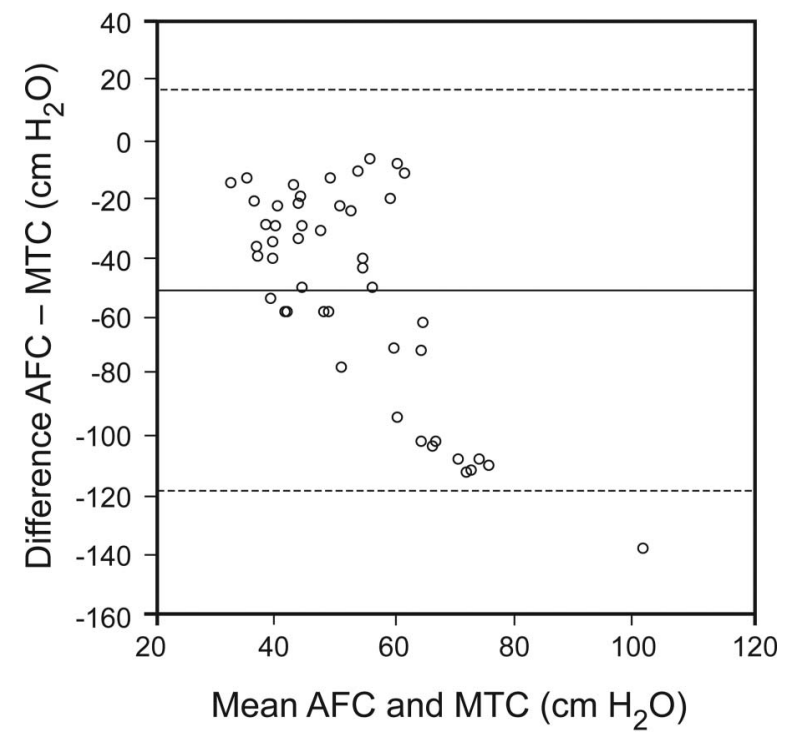

Fig. 4. In vivo esophageal pressure change during sniff maneuvers. $\mathrm{AFC}=$ air-filled balloon catheter, $\mathrm{MTC}=$ microtransducer catheter.

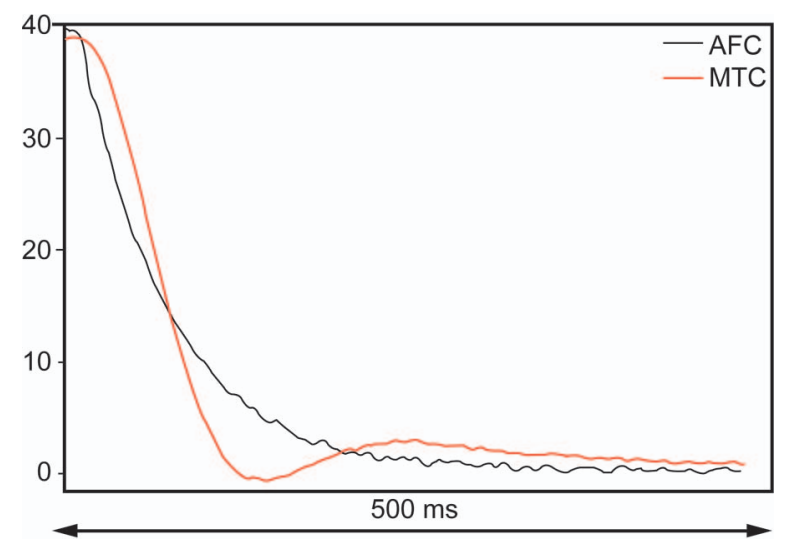

Fig. 5. Response time curves for the air-filled balloon catheter (AFC) and microtransducer catheter (MTC).

\section{In Vitro Linearity and Response Time}

The linearity of the microtransducer catheter amplifier outputs and air-filled balloon catheter pressure transducer was high and similar $(99.8 \% \pm 0.1$ for microtransducer vs $99.9 \pm 0.1 \%$ for air-filled balloon, $P<.99$ ). The microtransducer catheter had a response time significantly higher than the air-filled balloon catheter $(262 \times 114 \mathrm{~Hz}, P<.01)$ (Fig. 5).

\section{Discussion}

We showed, in an in vivo study, that the microtransducer catheter and the air-filled balloon catheter had a small and similar esophageal pressure drift over $3 \mathrm{~h}$. We also showed that the agreement between the catheters was poor for both baseline and esophageal pressure change measurements.

Interest in the clinical use of esophageal pressure to guide mechanical ventilation has increased. ${ }^{3}$ Recently, a report summarized the current technical knowledge about esophageal pressure measurements in patients receiving mechanical ventilation, but no recommendation was made regarding the type of esophageal catheter. ${ }^{1}$ Previously, in vitro and in vivo short-term studies compared the performance of different esophageal catheters. However, to be useful in the clinical arena, the performance of the esophageal catheters should be compared in long-term in vivo studies.

Three previous studies in vitro evaluated the baseline drift of the esophageal pressure and reached controversial results. The first study concluded that after a 16 -h period, no drift was observed in the microtransducer catheter. ${ }^{8}$ The second study concluded that there was a baseline drift over a 6-h period, that the drift could be in either a positive or negative direction, that it was not linear, and that most of the drift occurred during the first hour of use. ${ }^{6}$ Finally, the third study evaluated only the drift of the zero position and showed that there was a significant drift. ${ }^{14}$ The reasons for the discrepant results are not clear, since the calibration of the catheters, the procedures to guarantee the thermal stability, and the previous soaking of the catheters were similar among the studies.6,8,14 One reason for the difference could be the manufacturing of the catheters, since they were produced by different manufacturers. In our study, we employed a microtransducer catheter by the same manufacturer used in the studies that showed a significant drift. ${ }^{8} 14$ Because we took the same care about soaking, thermal stability, and calibration, we hypothesize that improvements in the design and manufacturing of the microtransducer catheter are responsible for the higher stability in the present study.

We considered that due to the microtransducer catheter advantages over the air-filled balloon catheter, the microtransducer catheter would be more suitable for mechanical ventilation guidance. This assumption led us to measure the agreement of a microtransducer catheter and an airfilled balloon catheter, to verify whether the microtransducer catheter would agree sufficiently to replace the airfilled balloon catheter. Four previous studies evaluated the agreement of absolute esophageal pressure values and changes of the esophageal pressure, again with discrepant results. Two studies showed an absolute pressure poor agreement but a good agreement in the pressure changes. ${ }^{6,8}$ A third study also concluded that the values of esophageal pressure changes measured by the microtransducer catheter were similar to those measured by the air-filled balloon catheter. However, in that study, the air-filled balloon catheter values were from historical controls. ${ }^{9}$ The last study ${ }^{14}$ 
showed, as we did, that the agreements for absolute esophageal pressure and for changes in esophageal pressure were poor. There are 2 reasons to explain the difference in the esophageal pressure agreement between the studies: first, the use of catheters of different manufacturers, and second, differences in the catheter placement, leading to different coupling of the microtransducer and the air-filled balloon. Besides the use of different catheters and different catheter placement, we believe that the microtransducer catheter and the air-filled balloon catheter have different characteristics that led to poor agreement. First, the frequency response of the microtransducer catheter is faster than that of the air-filled balloon catheter, so during changes in esophageal pressure, especially fast pressure changes, the microtransducer catheter would record greater changes in pressure. Second, microtransducer catheters record pressure at a single point so that the measured esophageal pressure may not be equal to the pressure measured by air-filled balloon catheters over a larger area. ${ }^{15}$ Third, microtransducers have a very small area, so adhesion of mucus, mechanical forces exerted by the catheter shaft, and the sticking of the sensor on the esophageal wall can exert van der Waals forces on the sensor, resulting in unpredictable extra stress and faulty measurement ${ }^{8,14} \mathrm{Fi}$ nally, due to the higher response time of the microtransducer catheter, the cardiac and swallowing artifacts are more frequent and intense and may merge with the esophageal pressure trace. According to the previous study and the manufacturer's instructions, we tried to reduce the baseline drift by soaking the microtransducer catheter in water for $1 \mathrm{~h}$ before use and reducing amplifier gain (in our case, we did not apply any gain). Probably due to those precautions, we obtained a good in vitro and in vivo stability. The air-filled balloon catheter had an in vivo baseline drift of approximately $-0.5 \mathrm{~cm} \mathrm{H}_{2} \mathrm{O}$, whereas the microtransducer catheter had a baseline drift of approximately $1.0 \mathrm{~cm} \mathrm{H}_{2} \mathrm{O}$ after $3 \mathrm{~h}$. Although statistically significant, that difference does not seem clinically relevant.

\section{Advantages and Disadvantages of the Microtransducer Catheter}

The air-filled balloon catheter has been used in clinical and experimental situations for a long time, and its advantages and disadvantages are well known.1,2,11 The interest is now in surrogates, such as the microtransducer catheters. One advantage of the microtransducer catheter is that it is more comfortable to pass through the nose, to swallow, and to be in contact with the nasopharynx and oropharynx, especially during a long hospital stay. We did not measure comfort objectively, but most of the volunteers considered the microtransducer catheter more comfortable to be inserted and to be kept in the right position for the 3-h period. Another advantage of the microtransducer cath- eters over the air-filled balloon catheters is that the microtransducer catheters are easier to place in the expected esophageal and gastric position. This advantage comes from the fact that the esophageal and gastric curves are displayed on a screen and in real-time from beginning to end of the placement of the catheter, so it is possible to know the position of the proximal and distal transducers every time. A final advantage is that there is no variation in the pressure measurement due to differences in the volume of air in the balloons or air leaks. Air-filled balloon catheters are greatly influenced by the volume of air in the balloons and the surrounding pressure, so the esophageal pressure can easily be under- or overestimated. ${ }^{7,16}$

On the other hand, the microtransducer catheters have some disadvantages. One disadvantage comes from the small size of the microtransducer, which is able to measure the pressure only of a very small area, which may not reflect the real esophageal pressure, since a long esophageal balloon is necessary to reflect the mean pressure along the esophagus. Another disadvantage may be the difficulty in inserting the microtransducer catheter in patients with an impaired swallowing capability, since a more rigid catheter may be required in such cases. Our experience with microtransducer catheters in intubated patients receiving mechanical ventilation showed us that they are more difficult to insert than the air-filled balloon catheters. A final disadvantage is that reference values established with airfilled balloon catheters may not be applicable to catheters with miniature pressure transducers.

\section{Limitations}

We hypothesized that microtransducer catheters could be more suitable than air-filled balloon catheters for use by patients receiving mechanical ventilation; however, due to limitations in testing the catheters in patients, as a first step, we decided to compare the catheters among healthy volunteers. First, almost all patients receiving mechanical ventilation use a large nasogastric tube, and the presence of 3 catheters in the esophagus could lead to spurious results. Second, the 2 catheters and the nasogastric tube would block both nostrils, increasing the chances of injuring the nostrils and blocking sinus drainage. Third, we had planned to test the agreement during deep inspirations (sniff maneuvers), and critically ill patients are not capable of volitional tests. However, we believe that the evaluation of esophageal pressure in volunteers in the semi-recumbent position is a good model to study patients receiving mechanical ventilation because the most relevant factor that affects esophageal monitoring in patients receiving mechanical ventilation is the semi-recumbent position due to the pressure vector generated by the weight of mediastinal content of the lung and thoracic cage, ${ }^{17}$ and we also studied the volunteers in a semi-recumbent position. Another 
factor that affects esophageal monitoring in patients receiving mechanical ventilation is the presence of asymmetrical lung disease, ${ }^{18}$ but due to the numerous different possible lung disease presentations, even a clinical study with patients receiving mechanical ventilation could not control for that variable.

Finally, one could argue that the presence of 2 catheters could make the results less reliable, but we believe that one esophageal catheter did not affect the other. First, the microtransducer catheter did not affect the air-filled balloon catheter because the air-filled balloon has a high compliance wall that could accommodate any other catheter leaning on it. As the most important evidence, it was demonstrated that even the presence of a thicker catheter, such as a nasogastric tube, did not invalidate the accuracy of esophageal pressure measurement, provided that the catheter is correctly positioned. ${ }^{19}$ Second, the air-filled balloon did not affect the microtransducer catheter because the air-filled balloon was filled with $4.0 \mathrm{~mL}$, and $3.5 \mathrm{~mL}$ were withdrawn, resulting in a filling volume of $0.5 \mathrm{~mL}$. Recently, Mojoli et al ${ }^{7}$ evaluated 6 different air-filled balloon catheters and showed that the catheter from Coopersurgical (the one that we employed) did not affect the surrounding pressure when filled from 0 to $2 \mathrm{~mL}$.

\section{Conclusions}

In vivo, the drift of the baseline esophageal pressure measured by the microtransducer catheter and by the airfilled balloon catheter are small and similar. The agreement of the microtransducer catheter and the air-filled balloon catheter is poor for measurements of absolute esophageal pressure and for changes in esophageal pressure. The differences in the performance between the microtransducer catheter and the air-filled balloon catheter preclude them from being used as surrogates and the normal ranges established with air-filled balloon catheters may not be strictly applicable.

\section{REFERENCES}

1. Akoumianaki E, Maggiore SM, Valenza F, Bellani G, Jubran A, Loring $\mathrm{SH}$, et al. The application of esophageal pressure measurement in patients with respiratory failure. Am J Respir Crit Care Med 2014;189(5):520-531.

2. Benditt JO. Esophageal and gastric pressure measurements. Respir Care 2005;50(1):68-75; discussion 75-77.
3. Talmor D, Sarge T, Malhotra A, O'Donnell CR, Ritz R, Lisbon A, et al. Mechanical ventilation guided by esophageal pressure in acute lung injury. N Engl J Med 2008;359(20):2095-2104.

4. Grasso S, Terragni P, Birocco A, Urbino R, Del Sorbo L, Filippini $\mathrm{C}$, et al. ECMO criteria for influenza A (H1N1)-associated ARDS: role of transpulmonary pressure. Intensive Care Med 2012;38(3): 395-403.

5. Beda A, Güldner A, Carvalho AR, Zin WA, Carvalho NC, Huhle R, et al. Liquid- and air-filled catheters without balloon as an alternative to the air-filled balloon catheter for measurement of esophageal pressure. PLoS One 2014;9(9):e103057.

6. Stell IM, Tompkins S, Lovell AT, Goldstone JC, Moxham J. An in vivo comparison of a catheter mounted pressure transducer system with conventional balloon catheters. Eur Respir J 1999;13(5):11581163.

7. Mojoli F, Chiumello D, Pozzi M, Algieri I, Bianzina S, Luoni S, et al. Esophageal pressure measurements under different conditions of intrathoracic pressure: an in vitro study of second generation balloon catheters. Minerva Anestesiol 2015;81(8):855-864.

8. Peters RJ, Meijer JH, Kingma R, Scheffer GJ, Heethaar RM. Evaluation of catheter-mounted transducers for intra-oesophageal pressure recording in respiratory function tests. Med Biol Eng Comput 1998;36(5):562-567.

9. Evans SA, Watson L, Cowley AJ, Johnston ID, Kinnear WJ. Normal range for transdiaphragmatic pressures during sniffs with catheter mounted transducers. Thorax 1993;48(7):750-753.

10. Panizza JA, Finucane KE. Comparison of balloon and transducer catheters for estimating lung elasticity. J Appl Physiol 1992;72(1): 231-235.

11. Mead J, McIlroy MB, Selverstone NJ, Kriete BC. Measurement of intraesophageal pressure. Journal of applied physiology 1955;7(5): 491-495.

12. Baydur A, Behrakis PK, Zin WA, Jaeger M, Milic-Emili J. A simple method for assessing the validity of the esophageal balloon technique. Am Rev Respir Dis 1982;126(5):788-791.

13. Bland JM, Altman DG. Statistical methods for assessing agreement between two methods of clinical measurement. Lancet 1986;1(8476): 307-310.

14. Beardsmore CS, Wong YC, Stocks J, Silverman M. Assessment of the catheter tip pressure transducer for use in infant lung function tests. Med Biol Eng Comput 1982;20(6):657-660.

15. American Thoracic Society/European Respiratory Society. ATS/ERS statement on respiratory muscle testing. Am J Respir Crit Care Med 2002;166(4):518-624.

16. Walterspacher S, Isaak L, Guttmann J, Kabitz HJ, Schumann S. Assessing respiratory function depends on mechanical characteristics of balloon catheters. Respir Care 2014;59(9):1345-1352.

17. Knowles JH, Hong SK, Rahn H. Possible errors using esophageal balloon in determination of pressure-volume characteristics of the lung and thoracic cage. J Appl Physiol 1959;14(4):525-530.

18. Graf J, Formenti P, Santos A, Gard K, Adams A, Tashjian J, et al. Pleural effusion complicates monitoring of respiratory mechanics. Crit Care Med 2011;39(10):2294-2299.

19. Niknam J, Chandra A, Adams AB, Nahum A, Ravenscraft SA, Marini JJ. Effect of a nasogastric tube on esophageal pressure measurement in normal adults. Chest 1994;106(1):137-141. 